\title{
Peculiarities of genome structure of flowering plants
}

\author{
I. Erokhin \\ NBK LLC, Moscow, Russia \\ e-mail:i.erokhin@inbox.ru
}

Key words: flowering plants, cereals, ontogenesis, junk DNA, Genome Tree Theory

The meristem of plants contains cells with different genomes. The genome of somatic cells differs from that of generative cell after crossing-over. The genome of zygote and embryo cells also differs from that of somatic cells. Some alleles of the polyploid endosperm cells genome, in its turn, differs from the haploid component of genomes of somatic cells, zygote or embryo cells. Despite the fact that the steps of Genome Tree [1], which determine the ontogenesis of apical generative cells and apical somatic cells are located on different branches of the Genome Tree (and may belong to somewhat different variants of the Genome Tree), these cells are located close to each other in apical meristem in terms of space. Moreover, in the process of branching, they are divided simultaneously and continue to stay close to each other in each new offshoot.

In the process of branching, the Genome Tree step of the mother somatic cell in apical meristem transfers the management in each daughter cell to the root (in terms of graphs theory) step of the Genome Tree branch responsible for offshoot ontogenesis. Such complete looping of the Genome Tree fragment may ensure "endless" plant growth. Similarly, the Genome Tree fragment of the mother generative cell in the apical meristem is also fully looped in the process of branching. Grafting a veneer that contains such an offshoot forms a whole new plant. Spontaneous mutations of regulatory non-coding genes that change the looped somatic branch of the Genome Tree change the phenotype of the plant. This property is the basis of fruit trees domestication. At the same time, generative branch remains unchanged and the plant that grows from the planted seed preserves the old unchanged phenotype. The mutations that change the generative branch of the Genome Tree do not change the current phenotype of the plant, but influence the phenotype of net generation plants that grow from the seeds.

If looping of the Genome Tree fragment is limited by the external loop that contains several steps, the plant growth will not be "endless". For example, a wheat stem consists of several metamers, and a head also contains several spikelets. Introduction of mutations that extend the external loop of the Genome Tree fragment, responsible for head formation, should lead to increase in the number of grains in the head and thus better wheat yield. Spontaneous mutations of non-coding regulatory genes may lead to appearance of plants of larger size. If such a phenotype change is associated with mutations in the somatic branch of the Genome Tree, the next generation of plants loses these beneficial properties. New properties can be saved in several ways. Modeling the structure of looped fragments and other branches of Genome Tree that are responsible for ontogenesis of separate organs and groups of plant cells is of great interest, but this is the subject of other studies.

\section{References}

1. Erokhin I. (2016) Genome tree theory. International Conference on the Mathematical Modeling and High-Performance Computing in Biomedicine and Biotechnology, August 29-September 2, Novosibirsk, Russia. 